\title{
Comparison of Different Regimens for Surgical Hand Preparation
}

T o reduce the potential risk of surgical site infections, surgical staff members perform a lengthy regimen of scrubbing with an antiseptic agent, usually detergent based, before participating in a surgical procedure. The frequent and prolonged use of water, a scrub brush or sponge, and the surfactants in antiseptic soaps and detergents contribute to deterioration of the hand's skin condition. ${ }^{.}$Resulting damage to the skin can lead to potentially undesirable changes in colonizing skin flora, such as increased colonization with gram-negative bacteria and Candida species. ${ }^{2}$

In addition to concerns about reducing risk of postoperative infections, hospitals also are concerned with the best use of staff member time and hospital resources. Evidence shows that providing health care professionals with an effective and safe waterless alternative to preoperative scrubs may conserve time and supplies. One group of researchers estimated that traditional hand washing consumes $80 \%$ more nursing staff member time than the use of an alcoholbased waterless hand degerming agent in intensive

\section{$\begin{array}{llllllll}A & B & S & T & R & A & C & T\end{array}$}

Twenty surgical staff members participated in a clinical trial to compare the microbiology and skin condition of hands when using a traditional surgical scrub (TSS) with a detergent-based antiseptic containing $4 \%$ chlorhexidine gluconate (CHG) and a short application without scrub of a waterless hand preparation (HP) containing $61 \%$ ethyl alcohol, $1 \% \mathrm{CHG}$, and emollients. The HP was associated with less skin damage $(P=.002)$ and lower microbial counts postscrub at days five $(P=.002)$ and $19(P=.02)$. The HP protocol had shorter contact time (HP mean $[M]=80.7$ seconds; TSS $M=144.9$ seconds; $P<.0001)$, and more subjects preferred the HP regimen $(P=.001)$. The HP performed better than the TSS, was less costly, and should be evaluated in larger trials and considered for widespread implementation. AORN J 73 (Feb 2001) 412-432. care units, but no studies have compared the time equirements of the traditional surgical scrub (TSS) waterless hand preparation (HP) in the OR. ce of this clinical trial, therefore, was to PS with a waterless alcohol-based HP

\section{METHODOLOGY}

This was a six-week, single center, prospective tive weeks and, after a one-week hiatus, used the TSS hree consecutive weeks. jujects were full-time surgical staff members who and met the following criteria:

had been using a traditional scrub product (ie, detergent-based containing $4 \%$ chlorhexidine gluconate $[\mathrm{CHG}]$ ) as their preoperative scrub for at least two weeks before enrollment;

- were expected to be available throughout the course of the study (ie, seven weeks);

- agreed not to use lotions on their hands during the course of the study, except when prescribed by study staff members; and

- were willing to comply with the requirements of the study and give informed consent.

Exclusion criteria included

- known allergy to any study material, 
- prolonged absence from work during the two weeks before the study or anticipated absence during the seven-week course of the study, and

- systemic antibiotic treatment during the course of the study.

The researchers determined sample size for the study on the desired difference to be detected in skin condition as measured by the visual scoring of skin (VSS) form. Previous studies estimated a standard deviation (SD) $\pm 1.3 .^{+}$To have $90 \%$ power to detect a difference of 1.0 on the VSS scale at the 0.05 level of significance, the study required at least 20 subjects. ${ }^{5}$ To account for potential dropouts and post-study exclusions, 27 OR staff members were enrolled and randomized by a computer-generated randomization

\section{Table 1}

VISUAL SCORING OF SKIW

A trained expert grader, using a $3 \times$ magnifying glass, visually inspected the skin on the dominant hand of participants using the following interpretive scale.

1 = Extensively scaly

- Extensive cracking of skin surface.

- In some cases, scales are very large, but some individuals never develop large scales.

- The skin is very irritated with widespread reddening and/or occasional bleeding.

\section{2 = Very scaly}

- More scale and pronounced separation of scale edges from skin, although they may still be lying flat on the skin surface.

- Some evidence of cracking in sulci and on plateaus.

- Skin may appear irritated with some reddening

$3=$ Scaly

- Visible scale giving the overall appearance of the skin surface a whitish appearance.

- Definite upliffing of edges or scale sections.

- Hand is rough to the touch.

4 = Slightly scaly

- Scale in sulci and on plateaus.

- More visible scale that is more uniformly distributed, but with no widespread upliffing.

$\mathbf{5}=$ Very slightly scaly

- Occasional scale that is not necessarily uniformly distributed.

$6=$ Normal

- No observable scale or irritation of any kind. scheme into two groups-a treatment group of 22 subjects and five subjects who served as a control group to determine whether changes could have occurred that were not associated with the regimens being tested (eg, changes in the weather).

Test protocols and products. Two scrub protocols were compared. The first was a traditional surgical hand scrub (ie, a detergent-based antiseptic containing $4 \% \mathrm{CHG}$ ), which was one of several antiseptic products used in the study institution. The second was an investigational waterless hand rinse product containing $61 \%$ ethyl alcohol wt/wt $1 \%$ CHG and emollients. A new drug application currently is under review by the US Food and Drug Administration (FDA) for this product. The protocol for this study was submitted to the FDA before the study began.

Instruments. Three tools to measure skin condition were used. Two were completed by expert graders-the VSS (Table 1) and the erythema grading scale (Table 2) - and one by the subjects-the hand skin assessment (HSA) form (Table 3). In previous studies, the VSS and HSA forms demonstrated levels of interrater reliability less than 0.90 and have been found to be highly correlated with objective measures of skin condition (ie, transepidermal water loss, shedding of corneocytes). ${ }^{6}$ Five members of the research team conducted skin assessments using the VSS and erythema grading scale. Before beginning the study, raters were trained so that interrater agreement within one point was reached in at least $90 \%$ of readings.

Three members of the study team made random observations of surgical scrub technique used by subjects ( $\mathrm{n}=31$ during HP, $\mathrm{n}=30$ during TSS). Items recorded on the observation form included whether nails were clean and short, whether jewelry was removed, and the number and types of deviations from the scrub protocol for both products. Components of the scrub, including actual contact time with the scrub product, were timed with a stopwatch.

Microbiologic techniques. Before the prescrub sampling, subjects cleansed their hands for approximately 10 seconds using plain, nonantimicrobial liquid soap to remove transient dirt and flora. A modified glove-juice technique was used for sampling. The subject inserted the dominant hand into a sterile polyethylene bag containing $50 \mathrm{~mL}$ of sampling

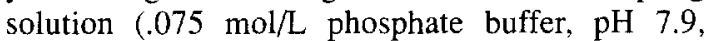
containing $3 \%$ polysorbate $80,0.1 \%$ t-Octylphenoxypolyethoxyethanol, and $0.3 \%$ lecithin). This solution 


\section{Table 2}

\section{ETITHEMA GRADING}

\section{A measure of skin condition completed by an expert} grader.

$0=$ Severe enthema covering a large area (>25\%) of the back of the hand, some areas may be intense in color, presence of edema or cracking

1 = Marked erythema, may include deeper areas, slight edema

$2=$ Moderate pinkness, more extensive in area (ie, $10 \%$ to $25 \%$ )

$3=$ Mild, diffuse erythema, limited to a small area (ie, $10 \%$ or less)

$4=$ Normal, no observable redness

neutralized any residual antiseptic on the skin and facilitated removal of microorganisms by dispersing the colonies into single cells, which then are counted as colony-forming units (CFUs). Pilot testing validated that adequacy and effectiveness of the sampling solution demonstrated no effect on the growth of the microorganisms and that the antiseptic was effectively neutralized.

The data collector massaged the entire hand through the wall of the bag for one minute and took samples to the microbiology laboratory within one hour for processing. An inoculum of $0.1 \mathrm{~mL}$ of sampling solution (undiluted, 1:10 and 1:100 dilutions of the glove juice solution) was plated, using a spread plate technique, onto the following agar media:

- sheep blood agar (5\%),

- MacConkey,

- colistin-nalidixic acid,

- Sabouraud's with chloramphenicol and gentamicin, - and bile esculin.

All plates were incubated at $35^{\circ} \mathrm{C}\left(\mathrm{ie}, 95^{\circ} \mathrm{F}\right)$ and observed daily for growth-more than 48 hours for bacteria and up to seven days for yeast. Using these dilutions, the countable range was 500 to $15,000,000$ CFUs per hand. Speciation of gram-negative bacilli was performed using API $20 \mathrm{NE}$ and Api 20E, staphylococci by Staphaurex, enterococci and micrococci by MicroScan Pos Combo Panel Type 12, and yeast by API 20 AUX and germ tube formation.

Procedures. The institutional review board of the study institution reviewed and approved the study. After recruitment, each subject signed a consent form, completed a demographic form, provided information regarding usual scrubbing practices and hand condition (eg, use of artificial nails, lotions, or polish, preexisting skin problems), was oriented to the study forms, was reminded to use the TSS product according to the manufacturer's directions (but was not specially trained in this technique because it was in current use in the study institution), and was trained in the application of the HP product (Table 4).

Skin condition assessments were obtained nine times. Both prescrub and postscrub cultures were

\section{Table 3}

HAND SKU ASSESSMIENT (COMPLATED BY SUBUECT)

On a scale of 1 to 7 , rate the current condition of your skin on both sides of your dominant hand.

\section{Appearance:}

Normal: no redness,

blotching, rash

$$
1
$$

3

4

Intactness:

Many abrasions or fissures

Moisture content:

Extremely dry

$$
1
$$

\section{Sensation:}

Extreme itching, burning, or soreness

$$
1
$$

3

4

(1)

Abnormal: red,

blotchy rash

7

Completely intact: no abrasions or fissures 7

Normal amount of moisture 7

No itching, burning, or soreness

3

$$
4
$$

5

6

7


obtained on days one and five of the first week and the last day of the third week for both regimens (ie, 13 cultures per subject, including baseline). Table 5 outlines the study schedule. Each subject kept daily pocket-sized diary cards to record the number of scrubs performed and the number of hours spent in surgery and wearing gloves. These were used to assess the potential confounding effects of gloving, number of scrubs, and hours in surgery on skin condition. Subjects randomized to the reference group completed the same skin assessment and daily diary cards as the study subjects, but did not have hand cul-

\section{Table 4}

\section{APPLIGATION REgIMIENS}

\section{A. Hand rinse}

1. Apply to clean, dry hands and nails; do not use a brush or sponge.

2. Dispense one pump (ie, $2 \mathrm{~mL}$ ) of alcohol product into the palm of one hand. Dip the fingertips of the opposite hand into the lotion and work it under the nails. Spread the remaining lotion over the hand and up the forearm.

3. Dispense another pump (ie, $2 \mathrm{~mL}$ ) of the product into the palm of the other hand. Dip the fingertips of the opposite hand into the lotion and work it under the nails. Spread the remaining lotion over the hand and up the forearm.

4. Dispense a final pump (ie, $2 \mathrm{~mL}$ ) of the lotion into the palm of either hand and reapply to both hands up to the wrist

5. Allow to dry before donning gloves.

\section{B. Traditional surgical scrub}

1. Open package. Wet hands and forearms to elbows with warm water.

2. Use nail cleaner to clean under the fingernails.

3. Wet the sponge and squeeze to work up a lather.

4. Scrub for three minutes, using the brush side of the product to clean nails, cuticles, and interdigital spaces. Use the brush side when scrubbing hands and forearms.

5. Rinse thoroughly with warm water.

6. Scrub for an additional three minutes, using the sponge side of the product only.

7. To produce additional lather, add a small amount of water to the sponge and squeeze.

8. Rinse hands and forearms thoroughly and dry with a sterile towel. tures obtained and did not participate in testing the two protocols. They continued to use the TSS throughout the entire study period.

To control for the potential confounding effects of lotion on skin condition, subjects agreed not to use hand lotion or emollients throughout the entire study, unless it was prescribed by project staff members. A CHG-compatible lotion was prescribed if the subject's VSS rating was two or lower (out of a possible six).

Throughout the course of the study, one or more members of the research team monitored compliance with study protocols, answered questions and concerns of subjects, and collected data daily in the OR. At the end of the study, subjects completed an exit questionnaire comparing the HP and TSS protocols for ease and speed of use, effect on skin and gloving, and preference.

Cost analysis. To compare the cost of the TSS and HP, the researchers obtained product costs from the manufacturers. Staff member time was calculated based on manufacturer instructions for the use of each product.

Data analysis. To assess skin condition, the researchers calculated a "change score" to compare the first and last ratings of the HSA, VSS, and erythema scales during the HP and TSS protocols. The change score controlled for differences among subjects in baseline skin condition. The Wilcoxon signed rank test was used to compare changes in skin condition ratings from baseline scores, and analysis of covariance (ANCOVA) was used to compare actual values with the baseline value as a covariate. Pearson product moment correlation coefficient was calculated to examine the relationship between total hours in surgery, total number of scrubs, total hours of glove wearing, and the HSA. Paired sample $t$ tests were used to compare mean hours of surgery and mean number of scrubs during the TSS and HP periods. Researchers used the Mann-Whitney $\mathrm{U}$ test to compare characteristics of the study and reference groups and a nonparametric test to compare scores between the TSS and HP periods to examine preferences of subjects for the two test regimens.

Counts of CFU were converted to $\log$ base $_{10}$ before statistical analysis to normalize the data. The primary microbiologic outcome of interest was the CFU per hand count at the end of each treatment period. First, paired $t$ tests were used to compare mean log CFU counts at each time interval between the HP and TSS protocols. Then an ANCOVA was used to compare postscrub counts between the two regimens at the end of test weeks one and three, controlling for prescrub counts.

\section{RESULTS}

Twenty-seven subjects initially were recruited. One subject developed skin redness during the HP test period and was removed from the study; a second 


\section{Table 5}

\begin{tabular}{|c|c|c|c|c|c|c|c|c|c|c|c|c|c|}
\hline & \multirow{4}{*}{$\begin{array}{l}\text { Initial } \\
\text { visit - } \\
\text { Friday }\end{array}$} & \multicolumn{12}{|c|}{ Treatment periods } \\
\hline & & \multicolumn{6}{|c|}{ Hand preparation } & \multicolumn{6}{|c|}{ Traditional surgical scrub } \\
\hline & & \multicolumn{2}{|c|}{ Week 1} & \multicolumn{2}{|c|}{ Week 2} & \multicolumn{2}{|c|}{ Week 3} & \multicolumn{2}{|c|}{ Week 4} & \multicolumn{2}{|c|}{ Week 5} & \multicolumn{2}{|c|}{ Week 6} \\
\hline & & Mon & Fri & Mon & Fri & Mon & Fri & Mon & Fri & Mon & Fri & Mon & Fri \\
\hline $\begin{array}{l}\text { Sign consent and } \\
\text { randomization }\end{array}$ & $\checkmark$ & & & & & & & & & & & & \\
\hline $\begin{array}{l}\text { Inclusion/exclusion } \\
\text { criteria }\end{array}$ & $\checkmark$ & & & & & & & & & & & & \\
\hline $\begin{array}{l}\text { Entrance } \\
\text { questionnaire }\end{array}$ & $\checkmark$ & & & & & & & & & & & & \\
\hline $\begin{array}{l}\text { Product training, } \\
\text { hand skin assessment } \\
\text { (HSA) orientation }\end{array}$ & $\checkmark$ & & & & & & & & & & & & \\
\hline $\begin{array}{l}\text { Visual scoring of } \\
\text { skin (morning before } \\
\text { first scrub) }\end{array}$ & $\checkmark$ & $\checkmark$ & $\checkmark$ & & $\checkmark$ & & $\checkmark$ & $\checkmark$ & $\checkmark$ & & $\checkmark$ & & $\checkmark$ \\
\hline $\begin{array}{l}\text { HSA (morning before } \\
\text { first scrub) }\end{array}$ & $\checkmark$ & $\checkmark$ & $\checkmark$ & & $\checkmark$ & & $\checkmark$ & $\checkmark$ & $\checkmark$ & & $\checkmark$ & & $\checkmark$ \\
\hline Prescrub hand culture & $\checkmark$ & $\checkmark$ & $\checkmark$ & & & & $\checkmark$ & $\checkmark$ & $\checkmark$ & & & & $\checkmark$ \\
\hline Test scrub & & $\therefore$ & $\checkmark$ & & & & $\checkmark$ & $\checkmark$ & $\checkmark$ & & & & $\checkmark$ \\
\hline $\begin{array}{l}\text { Postscrub hand } \\
\text { culture }\end{array}$ & & $\checkmark$ & $\checkmark$ & & & & $\checkmark$ & $\checkmark$ & $\checkmark$ & & & & $\checkmark$ \\
\hline \multicolumn{14}{|l|}{ Diany card (daily) } \\
\hline \multicolumn{14}{|l|}{$\begin{array}{l}\text { Scrub observations } \\
\text { ( } 30 \text { for each treat- } \\
\text { ment during weeks } \\
2,3,5 \text {, and } 6 \text { ) }\end{array}$} \\
\hline Exit questionnaire & & & & & & & & & & & & & $\checkmark$ \\
\hline
\end{tabular}

subject decided to discontinue participation after the first week. Hence, 25 subjects completed the study20 study subjects and five in the reference group (Table 6). There were no significant differences in demographic or practice variables (eg, age, years in current position, number of times gloved) between the reference and study groups (all $P>.65$ ). Five subjects were prescribed lotion during the HP period (two, however, had minimal skin damage but were adamant about requiring lotion), and six were prescribed lotion during the TSS period. During the HP period, significantly more hours were spent in surgery per person than during the TSS period (126.7 and 77.0 respectively, $P=.002$ ), but there was no significant difference in the reported number of scrubs ( 36.9 and 35.4 respectively, $P=.39$ ).
Mean baseline measurements of skin condition were 22.7 (out of maximum of 28) for HSA (range, 8 to $28 ; \mathrm{SD} \pm 4.6$ ), 4.50 (out of maximum of 6 ) for VSS (range, 2 to $6 ; \mathrm{SD} \pm 1.19$ ), and 3.1 (maximum $=4$ ) for erythema score (range, 2 to 4 ; $\mathrm{SD} \pm .85$ ). Mean $\log$ CFU count at baseline was 7.03 (range, 5.02 to 7.21 ; $\mathrm{SD} \pm .48$ ).

Microbiology. The majority of flora isolated were gram-positive cocci, including coagulase-negative staphylococci and micrococci, present in all positive cultures. There was one isolation of methicillinsensitive Staphylococcus aureus and 33 isolates of gram-negative bacteria. Most frequently isolated were Acinetobacter species (11, 33.3\%), Enterobacter species $(9,27.3 \%)$, and Klebsiella species $(6,18.2 \%)$. Two individuals had two gram-negative species 
isolated in a single sample. There also were 11 isolates of yeast, mostly Candida species $(9,81.8 \%)$. By the end of week one, the HP regimen was associated with significantly lower microbial counts than the TSS regimen (Figure 1). In the ANCOVA, the effects of treatment and subject were significant contributors to the model $(P=.04$ for both), while the effect of baseline microbial counts was insignificant (Table 7). The effect of baseline microbial counts was insignificant due to the inclusion of the subject effect in the model. The ANCOVA analysis was appropriate as the assumptions were well met; thus, the assertion can be made that there was a significant difference due to product in favor of the HP.

Skin condition. The reference group's VSS measurements revealed no mean changes greater than 0.6 and no observable trends; thus, sequential treatment periods were compared without taking environmental factors into account. The change scores for the VSS were significantly better during the HP protocol (changes from week one to week three-HP mean $[\mathrm{M}]=-0.4$; TSS $\mathrm{M}=-1.6 ; P=.002$ ). The ANCOVA, which included subject, product, and baseline VSS score as predictors of the week three VSS score, verified the aforementioned results, as product was found to be a significant contributor to the model $(P=.005)$. The ANCOVA analysis was appropriate because the assumptions were well met. Again, the self-assessment scores (TSS $M=21.2 ; \mathrm{HP} M=23.4$ ) and erythema ratings (TSS $\mathrm{M}=3.8 ; \mathrm{HP} \mathrm{M}=3.5$ ) were significantly better $(P=.046$ for TSS; $P=.008$ for HP) during the TSS regimen on the first day of the study only (Table 8 ).

Observation of technique. The direct contact time with the scrub product was significantly shorter for HP (M $=80.7$ seconds for HP; $M=144.9$ seconds for TSS; $P<.0001$ ). There were significantly more deficiencies in scrub technique during the TSS protocol (ie, $50 \%$ of the scrubs had at least one deficiency) than HP (ie, $6.5 \%$ had deficiencies; $P=.001$ ). Most of the deficiencies involved failure to clean fingernails and to scrub for the required amount of time. As a result, the total time required for hand preparation was not significantly different between the HP and TSS regimens (HP M $=199$ seconds; TSS $M=201.9$ seconds; $P=.87$ ).

Cost analysis. The direct costs of the two products are comparable. If manufacturer instructions are followed, the cost of regimen per application would be approximately $\$ 60$ for TSS and $\$ 20$ for HP (Table 9).

Subject preferences. Significantly more subjects preferred the HP regimen. They reported it as

\section{Table 6}

characteaistics of subJects

Characteristic Number

Surgical service

- Eye

- Cardiothoracic

$7(28 \%)$

- Neurosurger

$4(16 \%)$

- Orthopedic

- General and other

Primary shift

- Days

- Evenings or nights

Gender

- Female

- Male

Ethnicity

- Caucasian

$10(40 \%)$

- Hispanic

$5(20 \%)$

- Oriental

$7(28 \%)$

- African American

Position

- Surgical resident

- RN/licensed practical nurse

- Surgical technologist

- Physician assistant

Years in current position .02 to 19 years (mean $=6.4$ ) Age

23 to 52 years $($ mean $=36.1)$

being easier, faster, milder on hands, and conducive to donning gloves (Figure 2).

\section{DIScussion}

The practice among surgical staff members of scrubbing the hands before surgery has been highly regimented and ritualized. Surprisingly, however, with the exception of recommended practices published annually by AORN, there are few formal guidelines for surgical hand preparation.? For example, there currently are no guidelines listed under the topics of "surgical scrubbing" or "hand washing" in the National Guideline Clearinghouse. ${ }^{8}$ Increasingly, studies question certain practices such as timing of the scrub, use of a brush or sponge, and product selection. For example, one group of researchers demonstrated that a two-minute scrub with $4 \% \mathrm{CHG}$ was as effective at reducing bacterial growth as a threeminute scrub. ${ }^{9}$ Others have reported that a 30 -second wash with povidone-iodine was as effective as longer periods in reducing fingertip flora. ${ }^{10}$ In fact, one study reported that the hands of orthopedic surgeons 


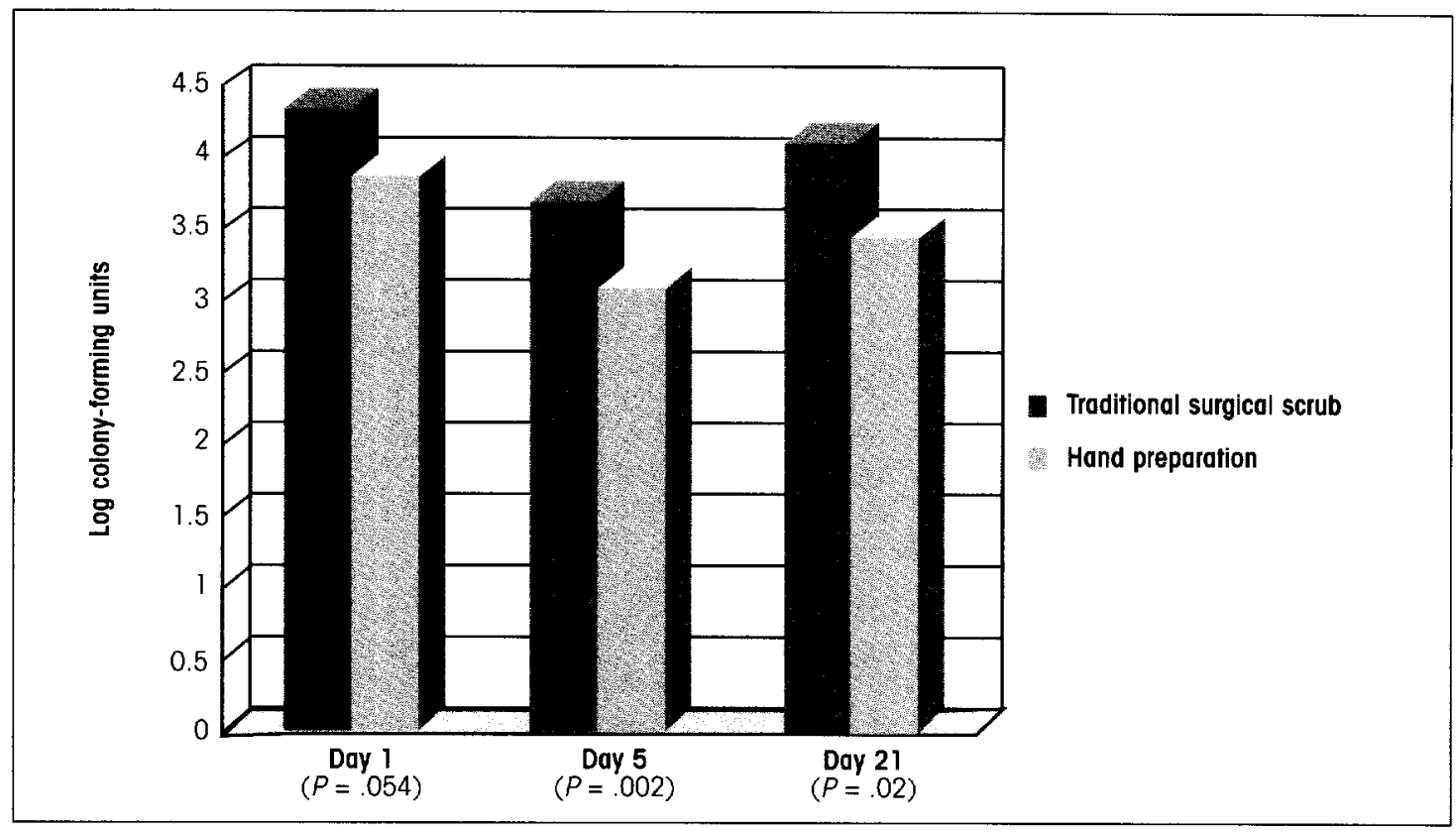

Figure 1 - Differences in postscrub log colony-forming units between traditional surgical scrub with chlorhexidine gluconate (CHG) detergent-based antiseptic and alcohol hand rinse containing $1 \% \mathrm{CHG}$. P values are from paired $t$ tests.

performing long procedures had significantly higher microbial counts after a 10 -minute scrub as compared to a five-minute scrub." The body of evidence continues to build that a lengthy scrubbing procedure is unnecessary.

Similarly, based on a growing data base, use of a brush or sponge appears to be counterproductive, causing skin damage and increased shedding. ${ }^{12}$ Several decades ago, researchers reported that microbial counts on hands were reduced satisfactorily by either a surgical scrub or an alcohol lotion, but that there was no increase in skin shedding after alcohol application as compared with an 18-fold increase after scrub.".? This is consistent with this study's findings that skin condition was rated to be significantly better during the HP protocol. The reduction in skin damage associated with a change from antiseptic detergent wash to alcohol lotion, plus the reduced shedding, led researchers to conclude that "a spirit based hand lotion should be used as a substitute for handwashing when this is done for degerming rather than cleaning."

Limitations. There were several limitations of this study. It was not possible to blind the product used, so subject and/or investigator bias cannot be ruled out, particularly for measures of skin health. We attempted to minimize this bias by giving the subjects careful instructions on completing the selfassessment form; using well-validated, standardized instruments; training subjects carefully; and testing interrater reliability. Subjects knew they were being observed and it is possible, therefore, that they modified their scrub practices. Further, although all subjects agreed to adhere to the test regimens, including not using lotion, there were deviations from the protocol. For some part of the trial period, both test products were available at the scrub sinks. To minimize this problem, one or more members of the research team were present for several hours each day (these hours varied, so that subjects did not know when researchers would be present) in the surgical suites to monitor protocol compliance, answer questions, and collect data. In fact, several deviations were noted by or reported to the research team, particularly with regard to the use of lotion. Fortunately, there was no evidence that these deviations were more common during one test period than another.

We were able to account for potential confounding effects due to frequency of scrubbing, hours in surgery, and hours of gloving in the data collection and analysis. Additionally, the fact that there were no changes in skin condition of hands of the reference group diminishes the likelihood that changes in outcome were associated with the passage of time rather than scrub regimen.

Microbiology. The excellent antimicrobial activity of alcohols has long been demonstrated, and alcohols 
Table 7

MICROBIAL COUNIS BY SCRUB REGIMIN

\begin{tabular}{|c|c|c|c|}
\hline \multirow[t]{2}{*}{ Time period } & \multicolumn{2}{|c|}{ Mean $\log _{10}$ counts ( \pm standard deviation) } & \multirow[t]{2}{*}{$P$ value (paired $t$ tests) } \\
\hline & $\begin{array}{l}\text { Hand preparation } \\
\text { regimen }\end{array}$ & $\begin{array}{l}\text { Traditional surgical } \\
\text { scrub regimen }\end{array}$ & \\
\hline Prescrub, week 1, day 1 & $4.80(.92)$ & $5.15(1.11)$ & .21 \\
\hline Postscrub, week 1, day 1 & $3.86(.84)$ & $4.34(1.24)$ & .054 \\
\hline Prescrub, week 1, day 5 & $4.32(.98)$ & $4.41(.53)$ & .67 \\
\hline Postscrub, week 1, day 5 & $3.09(.54)$ & $3.68(.83)$ & .002 \\
\hline Prescrub, week 3 , day 5 & $4.26(.86)$ & $4.64(.86)$ & .08 \\
\hline Postscrub, week 3, day 5 & $3.43(.98)$ & $4.09(1.29)$ & .02 \\
\hline
\end{tabular}

Table 8

CHANGES FROM BASELINE IN UISUAL SCORING OF SKIN* AS ASSESSED BY GXPERT GRADER

\begin{tabular}{|c|c|c|c|c|c|}
\hline \multirow[t]{3}{*}{ Time period } & \multicolumn{4}{|c|}{ Visual scoring of skin change from baseline } & \multirow[t]{3}{*}{$P$ value (paired $t$ tests) } \\
\hline & \multicolumn{2}{|c|}{$\begin{array}{l}\text { Hand preparation } \\
\text { regimen }\end{array}$} & \multicolumn{2}{|c|}{$\begin{array}{l}\text { Traditional surgical } \\
\text { scrub regimen }\end{array}$} & \\
\hline & VSS & $\mathbf{N}$ & VSS & $\mathbf{N}$ & \\
\hline Period baseline, week 1, day 1 & 4.7 & 22 & 5.1 & 19 & .094 \\
\hline Day 5 & -.2 & 20 & -8 & 18 & .148 \\
\hline Day 12 & -.4 & 19 & -1.2 & 18 & .011 \\
\hline Day 19 & -.4 & 19 & -1.6 & 16 & .002 \\
\hline
\end{tabular}

Range: $0=$ worst; $-6=$ best

are the gold standard for surgical hand preparation in several European countries; but in the United States, detergent-based antiseptics have been the standard for surgical scrubbing. ${ }^{15}$ Only recently have alcohol products been tested in this country for degerming hands of surgical staff members. Researchers reported no difference between a five-minute povidone-iodine scrub and a one-minute scrub followed by alcohol foam. ${ }^{16}$ Ethanol has been shown to be superior for killing superficial skin flora. Further, the combination of alcohol and CHG (as tested in this study) was even more effective at reducing counts of colonizing flora and producing residual antibacterial properties on the skin. ${ }^{17}$ Hence, the results of this study confirm previous studies and demonstrate the effectiveness of the alcohol/CHG combination when used in a preoperative surgical hand protocol.

Skin condition. The comparison of skin condition between products was not as rigorous as anticipated because some subjects scrubbed very few times per week and some participants were unavailable for a number of days during both phases due to nonstudy related work absences. This was a particular problem during the TSS testing phase; several subjects were unavailable for the final data collection points and subjects reported significantly fewer hours in surgery during that phase. Additionally, the weather during the seven-week trial period was unseasonably temperate; therefore, a type II error (ie, failing to detect differences in skin condition between the two treatments when they are present) is possible. As the skin condition results were weighted favorably toward the HP regimen (significantly better for some parameters), we postulate that there might have been even more striking differences if both products had been used in a more rigorous test-for more scrubs, over a longer period of time, or during harsh winter weather.

Although alcohols have been considered for decades to be the safest antiseptics available, there are rare accounts of urticaria syndromes and allergic contact dermatitis associated with alcohol. Similarly, anaphylactic reactions, keratitis, and other toxicities have been associated with $\mathrm{CHG}$. The incidence of such reactions has not been well described, but these phenomena deserve further investigation. ${ }^{18}$

Time and resource requirements. The contact time for the HP regimen was significantly shorter 


\title{
Traditional surgical scrub Hand preparation
}

\section{Product}

- Brush"

Price per case of preloaded scrub brushes

Number of brushes per case

Subtotal brush cost per scrub

$\begin{array}{cc}\$ 114 \text { to } \$ 150 & \text { N/A } \\ 300 & \text { N/A } \\ \$ 0.38 \text { to } \$ .050 & \text { N/A }\end{array}$

\section{- Solution}

Price per case of solution

Number of scrubs per case

Subtotal solution cost per scrub

\section{Staff member time}

Average length of scrubs or application" (including drying time)

Average cost of OR minute"

Subtotal cost of OR time per minute

\section{Total cost of product per application time}

\section{Additional costs for consideration}

- Weight of water waste per use

Empty $500 \mathrm{~mL}$ bottle

Nail cleaner

Used scrub brush and packaging

Subtotal weight of waste per use

\section{- Additional waste}

Water (2 gallons per minute flow rate)

Reusable sterile towel

$N / A$
$N / A$
$N / A$

6 minutes

$\$ 10$

$\$ 60$

$\$ 60.38$ to $\$ 60.50$

$\$ 256$ to $\$ 333$

1,600

$\$ 0.40$ to $\$ 0.52$

2 minutes

$\$ 10$

$\$ 20$

$\$ 20.40$ to $\$ 20.52$

\begin{abstract}
"Assumes staff members follow manufacturers' instructions for use (ie, no additional soap is used in conjunction with preloaded scrub brushes).

'Assumes staff members follow manufacturers' instructions for use (ie, the recommended three pumps of waterless product are used per application).

${ }^{7}$ At time of study, the waterless product was under US Food and Drug Administration review and not for sale. The price is an estimate.

'Manufocturers' directions for use for the chlorhexidine preloaded scrub brush require a total scrub time of six minutes.

"Application and dry time for the waterless product was observed to be approximately two minutes.

"Many institutions calculate the cost per minute for maintaining an active OR, including overhead and staff members. If available, this cost should be used.

\# Figure provided is per use weight (ie, $125 \mathrm{~g}$ for empty bottle divided by 80 uses per bottle).
\end{abstract}

than for the TSS. This difference would have been even more striking if subjects had scrubbed during the TSS phase for the six minutes specified by the manufacturer or the three to five minutes specified by the institution's protocol. In this study, the observed scrub time during the TSS was $M=146.6$ seconds. Both regimens required that nails be initially cleaned, but this frequently was not performed during the traditional scrub protocol. This accounted for the fact that there was no difference between the two regimens in total time used (ie, nails were cleaned before use of HP but not necessarily before the TSS). Hence, we conclude that the HP regimen could reduce time and resource requirements because there is a much shorter contact time needed and no need for running water or sterile towels to dry hands. The largest cost associated with scrubbing is personnel time rather than equipment; therefore, it should be noted that the cost analysis in Table 7 would vary considerably if OR staff members used either product for shorter or longer than directed. In this study, the alcohol regimen also resulted in fewer "errors" or deviations from protocol.

Conclusions and recommendations. In this 


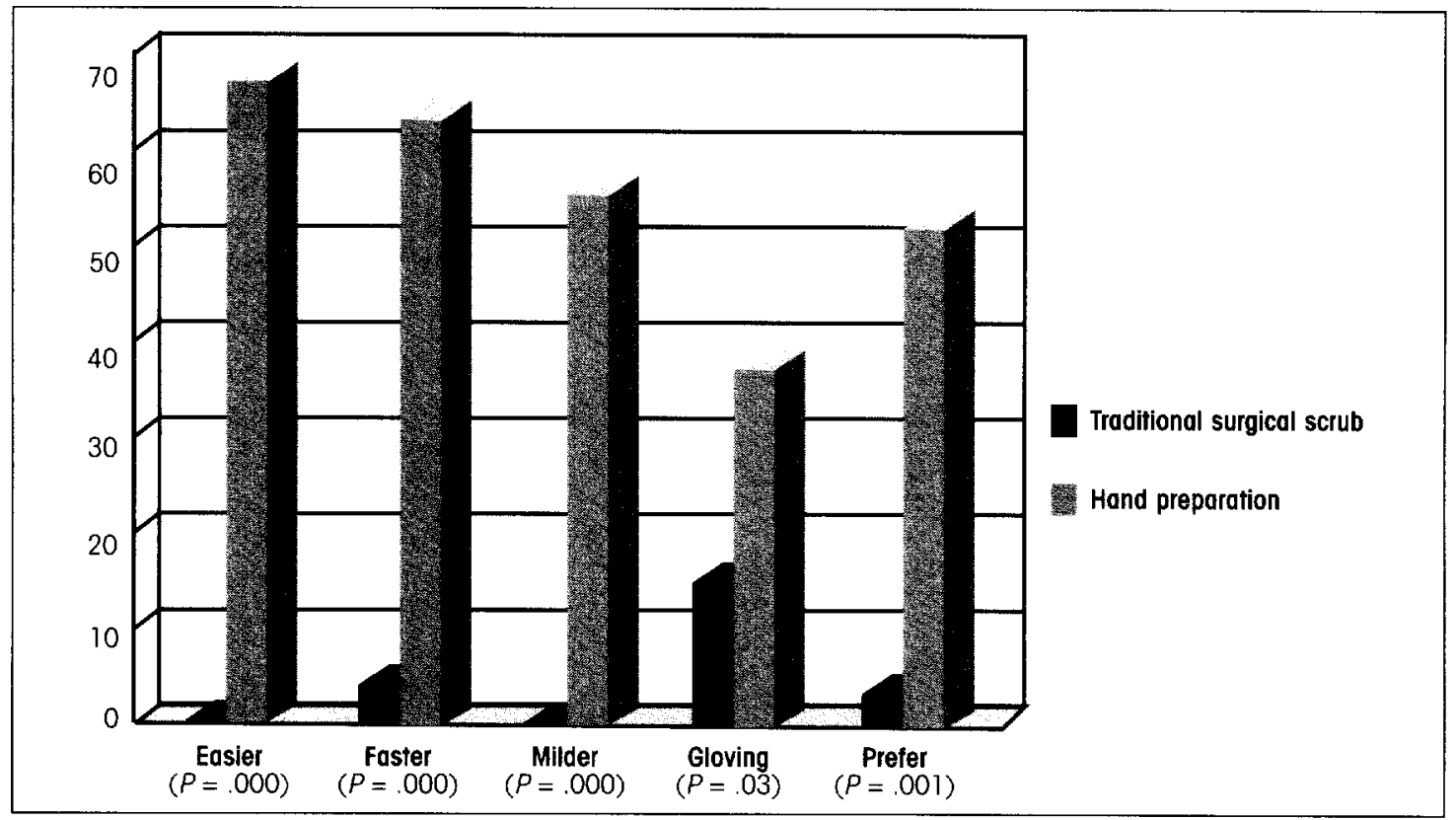

Figure 2 - Subject preferences between traditional surgical scrub and hand preparation.

clinical trial, a protocol for preoperative surgical hand preparation using a product containing $61 \%$ ethyl alcohol and $1 \%$ CHG resulted in significantly greater reductions in microbial counts on hands, improved skin health, and reduced time and resources. We recommend that such a combination of $\mathrm{CHG}$ and alcohol-based hand rinses be evaluated in larger trials and considered for OR use. In addition, we recommend current protocols that include the use of scrub brushes and scrubbing for prolonged periods of time be reassessed.

Elaine L. Larson, RN, PhD, CIC, FAAN, is a professor of pharmaceutical and therapeutic research, Columbia University School of Nursing, New York.

Allison $\boldsymbol{E}$. Aiello, $M S P H$, is a graduate research assistant, Columbia University Mailman School of Public Health, New York.

James $M$. Heilman, $B S$, is a clinical research specialist, $3 M$ Health Care, St Paul.
Christopher T. Lyle, MS, is a biostatistician, $3 M$ Health Care, St Paul.

Alicia Cronquist, $R N, B S N, M P H$, is a graduate research assistant, Columbia University Mailman School of Public Health, New York.

Julie B. Stahl, BS, is a clinical microbiology specialist, $3 M$ Health Care. St Paul.

Phyllis Della-Latta, PhD, FAAM, is an associate professor in the department of clinical pathology and medicine, Columbia University Medical Center, New York.

The authors gratefully acknowledge the assistance of Milysa Duong, BS, and Lena Lai, BS, during data collection; the facilitation of Kathleen Jakob, RN, BSN; the collaboration of the department of surgery, the nursing management, and staff members in the ORs, New York Presbyterian Medical Center; and the participation of so many $O R$ professionals.

Editor's Note: This research was funded in part by $3 \mathrm{M}$ Health Care, St Paul.

\section{NOTES}

1. A Jarrett, The Sweat Glands, Skin Permeation. Lymphatics, the Nails (New York: Academic Press, 1978) 1747; J E Kirk, "Hand washing. Quantitative studies on skin lipid removal by soaps and detergents based on 1500 experiments," Acta DermatoVenereologica Supplementum (Stockh) (1966) 1-183; J V Klauder, B A Gross, "Actual causes of certain occupational dermatoses III. A further study with special reference to effect of alkali on the skin, effect of soap on $\mathrm{pH}$ of skin, modern cutaneous detergents." Archives of Dermatology and Syphilology 63 (January 1951) 1-23.

2. E Larson et al, "Physiologic 
FEBRUARY 2001, VOL 73, NO 2

- Larson - Aiello - Heilman - Lyle •

- Cronquist - Stahl - Della-Latta -

and microbiologic changes in skin related to frequent handwashing," Infection Control 7 (February 1986) 59-63; E Larson et al, "Prevalence and correlates of skin damage on the hands of nurses," Heart and Lung 26 (September/October 1997) 404-412; E L Larson et al, "Changes in bacterial flora associated with skin damage on hands of health care personnel," American Journal of Infection Control 26 (October 1998) 513-521.

3. A Voss, A F Widmer, "No time for handwashing!? Handwashing versus alcoholic rub: Can we afford $100 \%$ compliance?" Infection Control and Hospital Epidemiology 18 (March 1997) 205-208.

4. E L Larson et al, "Differences in skin flora between inpatients and chronically ill outpatients," Heart \& Lung 29 (July/August 2000) 298305; Larson et al, "Physiologic and microbiologic changes in skin related to frequent handwashing," 59-63; Larson et al, "Prevalence and correlates of skin damage on the hands of nurses," 404-412; Larson et al, "Changes in bacterial flora associated with skin damage on hands of health care personnel," 513-521.

5. J H Zar, Biostatistical

Analysis, second ed (Englewood Cliffs, NJ: Prentice Hall, 1984) 153.

6. D R Highley et al, "A stereomicroscopic method for the determination of moisturizing efficacy in humans," Journal of The Society of Cosmetic Chemists 27 (August 1976) 351-363; Larson et al, "Physiologic and microbiologic changes in skin related to frequent handwashing," 59-63; Larson et al, "Prevalence and correlates of skin damage on the hands of nurses," 404-412.

7. "Recommended practices for surgical hand scrubs," in $A O R N$ Standards, Recommended Practices, and Guidelines (Denver: AORN, Inc, 2000) 271-276.

8. "National guideline clearinghouse." Available from http://www guideline.gov. Accessed 29 Aug 2000.

9. S M Wheelock, S Lookinland,

"Effect of surgical hand scrub time on subsequent bacterial growth," AORN Joumal 65 (June 1997) 1087-1098.

10. C Poon et al, "Studies of the surgical scrub," Australian and New Zealand Joumal of Surgery 68
(January 1998) 65-67.

11. D A O'Farrell et al, "Evaluation of the optimal handscrub duration prior to total hip arthroplasty," Journal of Hospital Infection 26 (February 1994) 93-98.

12. D W Hobson et al,

"Development and evaluation of a new alcohol-based surgical hand scrub formulation with persistent antimicrobial characteristics and brushless application," American Journal of Infection Control 26 (October 1998)

507-512; K Kikuchi-Numagami et al, "Irritancy of scrubbing up for surgery with or without a brush," Acta Dermato-Venereologica 79 (May 1999) 230-232; A F Peterson, "The microbiology of the hands: Effects of varying scrub procedures and times," in Volume 19, Development in Industrial Microbiology, Proceedings of the Thirty-Fourth General Meeting of the Society for Industrial Microbiology, East Lansing, Mich, Aug 21-26, 1977.

13. P D Meers, G A Yeo, "Shedding of bacteria and skin squames after handwashing," Journal of Hygiene London 81 (August 1978) 99-105.

14. P D Meers, "The shedding of bacteria and skin squames after handwashing," in Problems in the Control of Hospital Infection, ed S W B Newsom, A D S Caldwell (London: Royal Society of Medicine, 1980) 18.

15. H Beeuwkes, S H de Rooij, "Microbiological tests on operatingtheatre staff of a new disinfectant foam based on $1 \%$ chlorhexidine gluconate," Journal of Hospital Infection 8 (September 1986) 200-202; G

Kampf, M Hofer, C Wendt, "Efficacy of hand disinfectants against vancomycin-resistant enterococci in vitro," Journal of Hospital Infection 42 (June 1999) 143-150; H Kjolen, B $\mathrm{M}$ Andersen, "Handwashing and disinfection of heavily contaminated hands-effective or ineffective?" Journal of Hospital Infection 21 (May 1992) 61-71; J A Murie, S G Macpherson, "Chlorhexidine in methanol for the preoperative cleansing of surgeons' hands: A clinical trial," Scottish Medical Journal 25 (October 1980) 309-311; L J Pereira, G M Lee, K J Wade, "An evaluation of five protocols for surgical hand- washing in relation to skin condition and microbial counts," Journal of Hospital Infection 36 (May 1997) 4965; M E Reverdy, A Martra, J Fleurette, "Effectiveness of nine soaps and/or antiseptics on hand flora after surgical-type washing,"

Pathologie Biologie (Paris) 32 (June 1984) 591-595; K Tanaka et al,

"Evaluation of rapid drying hand disinfectant preparations in the intensive care unit," Critical Care Medicine 16 (May 1988) 540-542; M Zaragoza et al, "Handwashing with soap or alcoholic solutions? A randomized clinical trial of its effectiveness," American Journal of Infection Control 27 (June 1999) 258-26; M L Rotter, "Hygienic hand disinfection," Infection Control 5 (January 1984) 18-22.

16. N Deshmukh, J W Kramer, S I Kjellberg, "A comparison of fiveminute povidone-iodine scrub and one-minute povidone-iodine scrub followed by alcohol foam," Military Medicine 163 (March 1998) 145-147.

17. R Hall, "Degerming the hands of surgeons and nurses," in Problems in the Control of Hospital Infection, ed S W B Newsom, A D S Caldwell, (London: Royal Society of Medicine, 1980) 29-38.

18. E L Larson, H E Morton, "Alcohols," in Disinfection, Sterilization, and Preservation, fourth ed, $S$ S Block, ed (Philadelphia: Lea \& Febiger, 1991) 191-203; A Rilliet, N Hunziker, R Brun, "Alcohol contact urticaria syndrome (immediate-type hypersensitivity). Case report," Dermatologica 161 (1980) 361-364; H Okazawa et al, "Allergic contact dermatitis due to ethyl alcohol," Contact Dermatitis 38 (April 1998) 233; M Sticherling, J Brasch, "Alcohol: Intolerance syndromes, urticarial and anaphylactoid reactions," Clinics in Dermatology 17 (July/August 1999) 417-422; L M Hamed et al, "Hibiclens keratitis," American Journal of Ophthalmology 104 (July 1987) 5056; G T Layton, D R Stanworth, H E Amos, "The incidence of IgE and IgG antibodies to chlorhexidine," Clinical and Experimental Allergy 19 (May 1989) 307-314; M Okano et al, "Anaphylactic symptoms due to chlorhexidine gluconate," Archives of Dermatology 125 (January 1989) 50-52. 